\title{
Study on Rod Position Indication System using Permanent Magnets with Shielding Plates for a Control Rod Drive Mechanism
}

\author{
Jae Seon Lee ${ }^{1 *}$, Sang Soon Cho ${ }^{1}$, and Jong Wook Kim ${ }^{1}$ \\ ${ }^{1}$ Korea Atomic Energy Research Institute, Daedeok-daero 989-111, Yuseong-gu, Daejeon 34057, Korea
}

(Received 25 September 2015, Received in final form 2 December 2015, Accepted 2 December 2015)

\begin{abstract}
A control rod drive mechanism (CRDM) is an electromechanical equipment that provides linear movement for the control rods to control the nuclear reactivity in a nuclear reactor. A rod position indication system (RPIS) detects the control rod's position. To enhance the measurement accuracy of the system, a magnetostrictive type sensor with capability of generating operation limiting signals would be adapted instead of a conventional RPIS for a CRDM. An RPIS was modelled for a numerical analysis with the permanent magnets at the stationary limit positions and magnetic shielding plates with a moving permanent magnet. The performance analysis of the RPIS were conducted, and the results were discussed here.
\end{abstract}

Keywords : control rod drive mechanism, rod position indication system, magnetostrictive type sensor, magnetic shielding plates

\section{Introduction}

A control rod drive mechanism (CRDM) is an electromechanical device that provides linear movement to the control rods in order to control the nuclear reactivity in a nuclear reactor. A magnetic jack type CRDM consists of a motor assembly (including the latch assemblies), a pressure housing assembly, an extension shaft assembly (ESA), and a magnetic assembly (including lift and latch coils), as shown in Fig. 1. The performance of this type of device has been proven to be highly reliable over the last several decades based on the operation history of many commercial nuclear reactors, and the SMART (Systemintegrated Modular $A$ dvanced ReacTor, a Korean small and medium-sized reactor) adopts its basic mechanism. The nuclear reactivity depends on the vertical position of the control rods, and the CRDM reads the control rod's position from the top of an extension shaft, as shown in Fig. 1. In general, an ESA connects a CRDM which is installed on the reactor head with the control rod assembly. A rod position indication system (RPIS), mostly a reed switch type [1] or a discrete coil type [2], detects the vertical position of an ESA which represents the relative

CThe Korean Magnetics Society. All rights reserved.

*Corresponding author: Tel: +82-42-868-2826

Fax:+82-42-868-8188, e-mail: leejs@kaeri.re.kr position of the control rods, and rough accuracy has been acceptable for a commercial nuclear reactor; however, the higher accuracy is required for the SMART because the nuclear core is more sensitive to the movement of control rods than a commercial nuclear reactor core. A magnetostrictive type sensor would be an alternative as an accurate RPIS. Magnetostriction means deformation of ferromagnetic material due to the magnetostrictive strain from the applied magnetic field [3], and this can read multi-position at the same time. There are several information using a magnetostrictive type RPIS [4, 5]; however, they do not provide the electrical limit signals. An Upper electrical Limit (UEL) and a Low Electrical Limit (LEL) signal provide the upper and lower driving limits of an ESA to assure the operating safety of the CRDM and the reactor. Lee [6] applied a patent for an RPIS with capability of sensing limit positions based on the capability of multi-point reading of a magnetostrictive type sensor. This proposal has not been verified, so the function needs to be proved before developed. Because RPISs are disposed outside of a nuclear reactor pressure boundary, a permanent magnet inside of a pressure boundary delivers the representative position of an ESA. A magnetic field broadens when it comes to an RPIS, the measuring resolution becomes deteriorated. To improve resolution of limiting position, the magnetic shielding plates $[7,8]$ can be considered. 


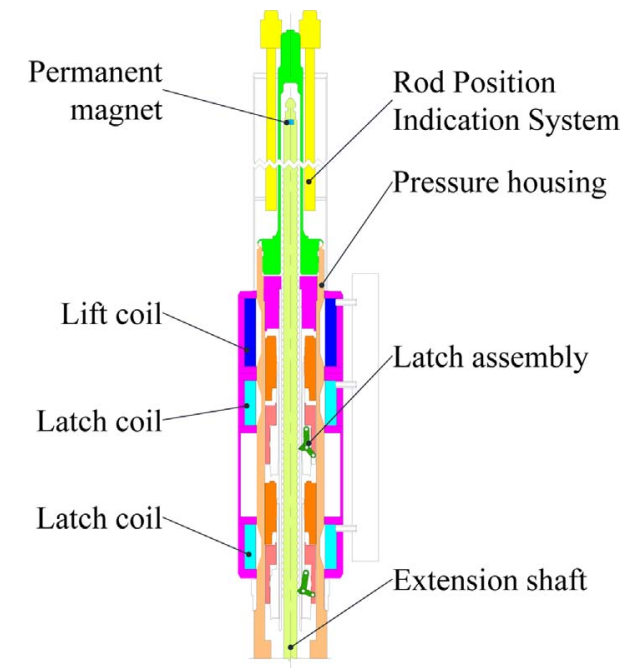

Fig. 1. (Color online) Schematic diagram of the CRDM.

In this paper, the analysis results of the magnetostrictive type RPIS applicability providing the electrical limit signals and the effect of the magnetic shielding plates are summarized.

\section{Analytical Approaching Method}

It is assumed that magnetostrictive wire made of nickel is installed in the center of an RPIS housing and $2 \mathrm{~A}$ of direct current pulses are flowing through. It is protected by a non-magnetic RPIS housing and disposed outside of an upper pressure housing. A permanent magnet, which represents the relative position of a control rod assembly and is assumed to be made of $\mathrm{NdFe} 30$, is transversing along with an ESA inside of an upper pressure housing. An electromagnetic field, which is developed by a direct current pulse passing through magnetostrictive wire, and a magnetic field surrounding a permanent magnet interfere with each other and bring about magnetostrictive strain in magnetostrictive wire. The induced elastic wave transfers to the sensing elements, and the elapsed time between the start and the return pulse is converted into distance to the permanent magnet. Because magnetostrictive strain may depend on magnetic flux density on the wire, it is necessary to estimate magnetic flux density on the wire at the initial stage. The electromagnetic analyses are performed to estimate magnetic flux density along magnetostrictive wire with Ansoft Maxwell ver. 14.0.0 [9].

Fig. 2 shows the simple 3D model considering only one of the electrical limit signal generating part of RPISs. A stationary permanent magnet is installed at the bottom of a nonmagnetic magnetostrictive wire protection housing

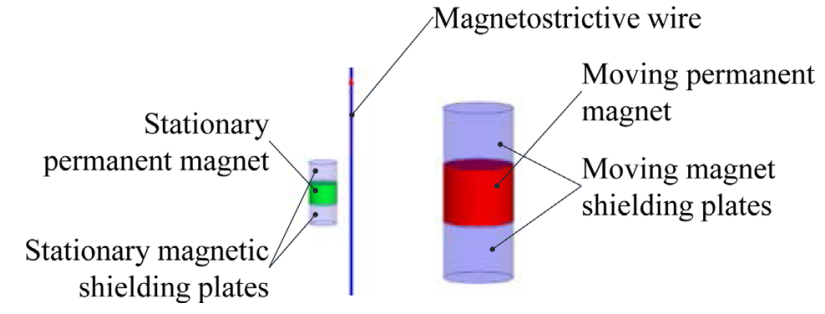

Fig. 2. (Color online) The simple analysis model.

closely at the opposite side of a moving permanent magnet. A moving permanent magnet moves up and down along with an ESA inside of an upper pressure housing, and the stroke is $2,000 \mathrm{~mm}$. The diameters of the moving and stationary permanent magnet are $25 \mathrm{~mm}$ and $10 \mathrm{~mm}$, respectively, and the heights are $20 \mathrm{~mm}$ and 7 $\mathrm{mm}$, respectively. The magnetostrictive wire center is 45 $\mathrm{mm}$ apart from the moving permanent magnet center and $10 \mathrm{~mm}$ from the stationary permanent magnet center. Magnetic shielding plates with the same diameter as the permanent magnet are positioned at each side of a permanent magnet to concentrate the magnetic field in the analysis. It is known that a wider plate is efficient to shield and concentrate the magnetic field [10]. However, the magnetic shielding plates for a CRDM cannot be much wider than the permanent magnet because the plates may prohibit coolant flow from passing through around them when the ESA drops for scram. So the same diameter as the permanent magnet is considered for the magnetic shielding plates in this study. The effect of the plate thickness will be discussed.

\section{Analysis Results}

Fig. 3 shows the resultant magnetic flux on magnetostrictive wire when the moving magnet travels upward with respect to the stationary magnet. The magnetic flux is maximized when two permanent magnets are at the same height, and it is getting diminished to the value from each permanent magnet along with a rising of the moving permanent magnet. When the moving permanent magnet moves up over $50 \mathrm{~mm}$ from the stationary magnet, two distinct magnetic flux peaks can be read. If the threshold levels of position detection are set as $10 \mathrm{~T}$ and $4 \mathrm{~T}$, the RPIS reads one position when two permanent magnets are at the same height and two different positions when the moving permanent magnet moves up $50 \mathrm{~mm}$. So it is possible to set the low limit of a control rod insertion or withdrawal within an accuracy range of \pm 50 mm. Fig. 4(a) and (b) show the resultant magnetic flux density distribution on the plane around the permanent 


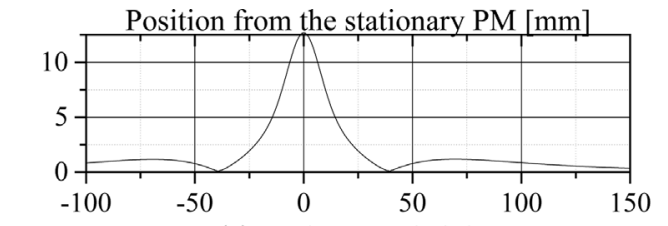

(a) At the same height

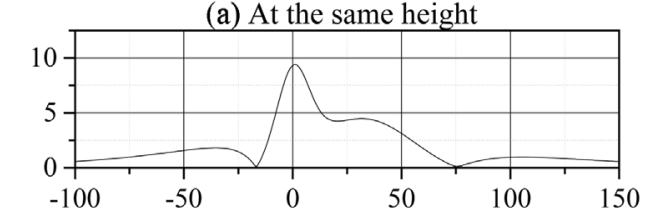

(b) Moving up $30 \mathrm{~mm}$

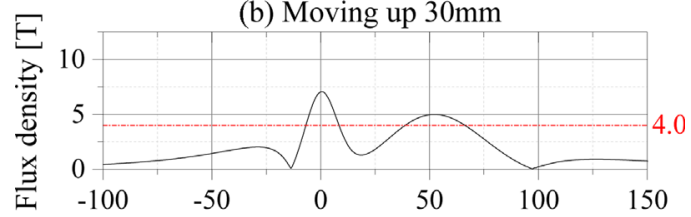

(c) Moving up $50 \mathrm{~mm}$

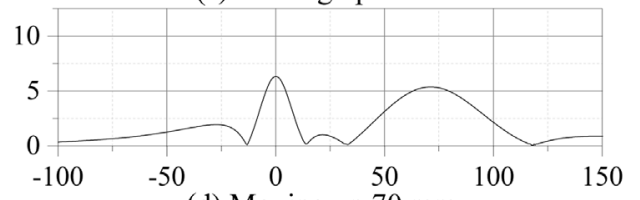

(d) Moving up $70 \mathrm{~mm}$

Fig. 3. (Color online) Resultant magnetic flux density changes according to the position of the moving permanent magnet.

magnets and the magnetostrictive wire when the moving permanent magnet and the stationary permanent magnet are at the same height and $50 \mathrm{~mm}$ apart, respectively.

The results show unrealistically high magnetic flux density along magnetostrictive wire because the wire is assumed to be ideal nickel which has relative permeability as 600 . However, the value would be much lower when the practical high-strain material like Terfenol-D is applied. Relative permeability of Terfenol-D is reported as 2 to 10 [11], however it depends on the production process and real values are not certain at this stage. Magnetic properties of high-strain wire shall be examined through dedicated magnetic property tests at the detailed design stage. Though the magnetic properties of highstrain material are not certain at this stage, the analysis results with Terfenol-D magnetostrictive wire with relative permeability as 10 are compared in Fig. 5. It shows that the maximum magnetic flux density will be $330 \mathrm{mT}$ when moving and stationary permanent magnets are at the same height. It shows the same tendency as the results in Fig. 3. Further research with the exact material properties will be performed at the detailed design stage.

Fig. 6 shows the resultant magnetic flux density according to the magnetized direction of the stationary and the moving permanent magnets. The permanent magnets are magnetized in the vertical direction. When each permanent

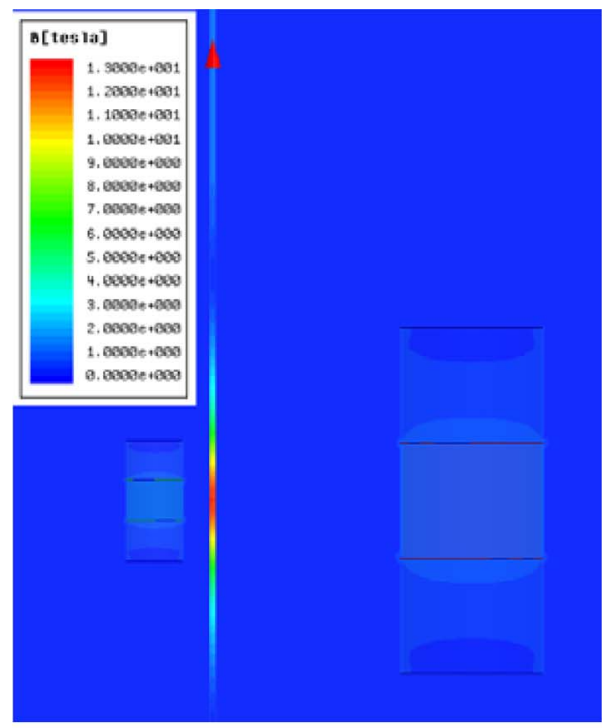

(a) For the case of Fig. 3(a)

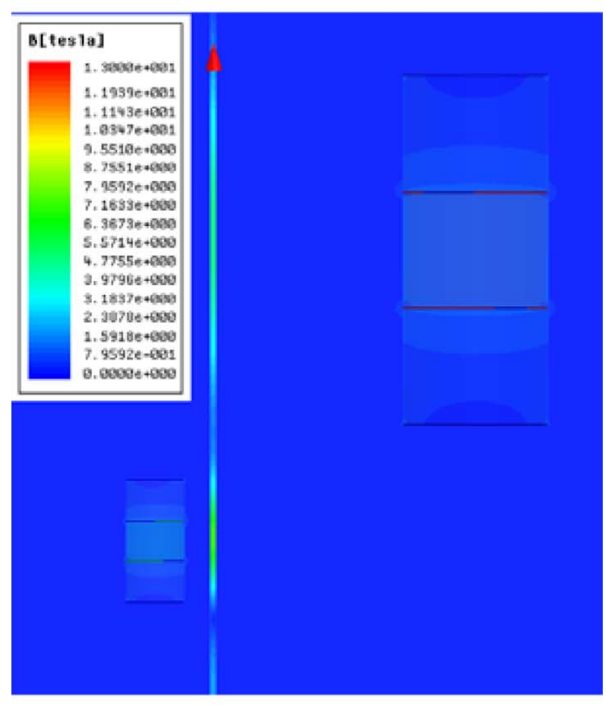

(b) For the case of Fig. 3(c)

Fig. 4. (Color online) Resultant Magnetic flux density distribution.

magnet is magnetized in the opposite direction, the magnetic fields cancel each other, as shown in Fig. 6(a) when they are at the same height. On the contrary, the magnetic field shows constructive interference when the magnets are magnetized in one direction, as shown in Fig. 5(a). However, when the moving permanent magnet moves up $50 \mathrm{~mm}$, magnetic flux density distribution is more distinguishable as shown in Fig. 6(b). To effectively distinguish the limit position, the permanent magnets magnetized in the same direction shall be installed. Fig. 7 shows resultant magnetic flux density distribution on the plane when each permanent magnet is magnetized in the opposite direction. Because of cancellation of magnetic 

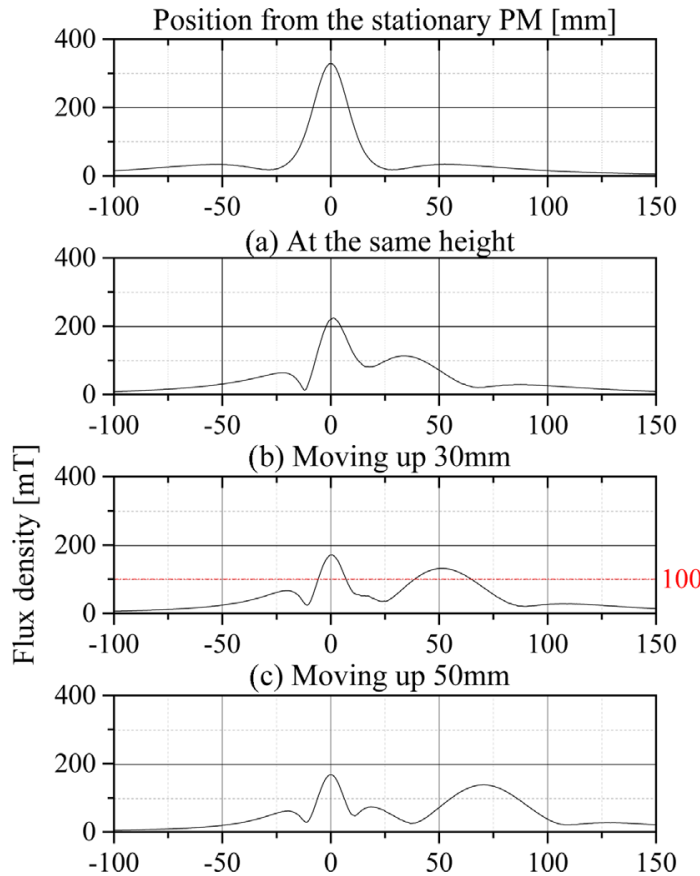

(d) Moving up $70 \mathrm{~mm}$

Fig. 5. (Color online) Resultant magnetic flux density changes with high-strain wire according to the position of the moving permanent magnet.

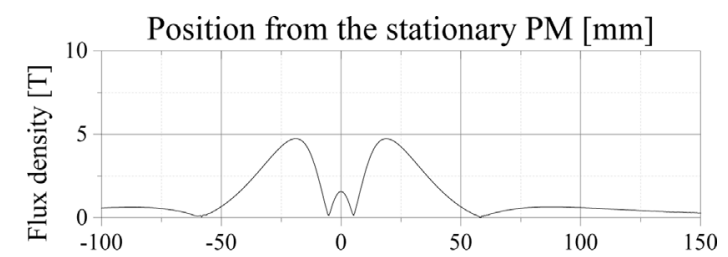

(a) At the same height

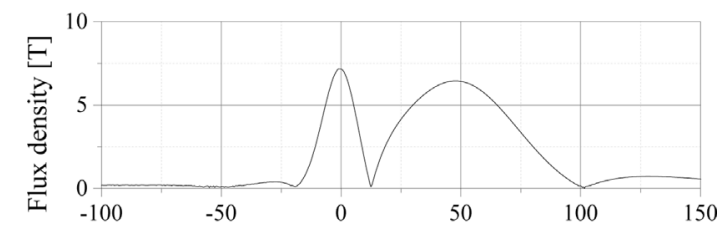

(b) Moving up $50 \mathrm{~mm}$

Fig. 6. Resultant magnetic flux density according to the magnetized direction.

fields, magnetic flux is getting diminished on the magnetostrictive wire at the height of the stationary permanent magnet.

Fig. 8 shows the effect of size of magnetic shielding plates when the stationary and the moving permanent magnets are at the same height. It is proved that the magnetic shielding plate contributes to enhance the position resolution of an RPIS. The highest magnetic flux density with $60 \mathrm{~mm}$ thick shielding plates increase by $28.8 \%$ over the result without the magnetic shielding

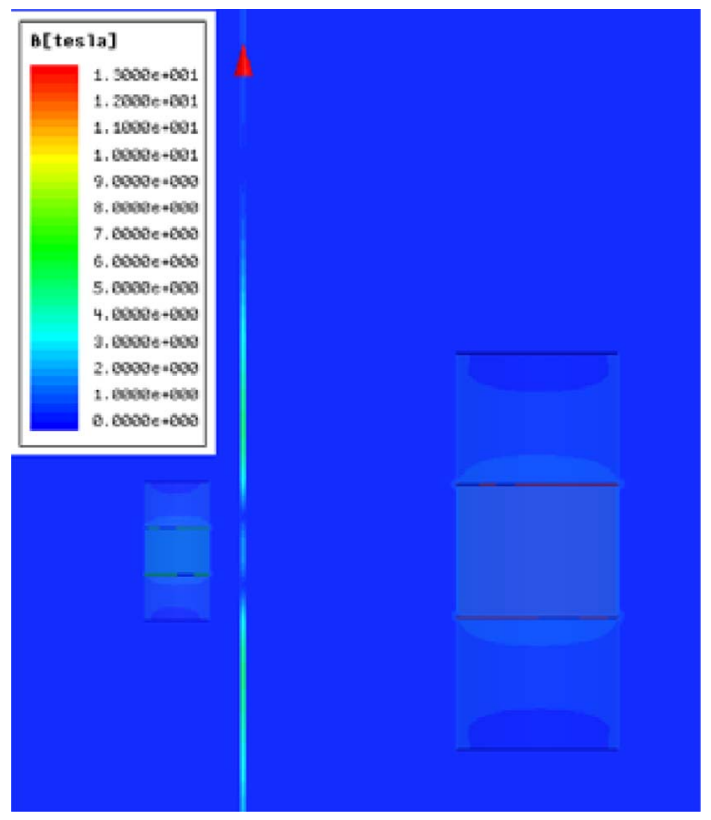

Fig. 7. (Color online) Resultant magnetic flux density distribution when each permanent magnet is magnetized in the opposite direction.

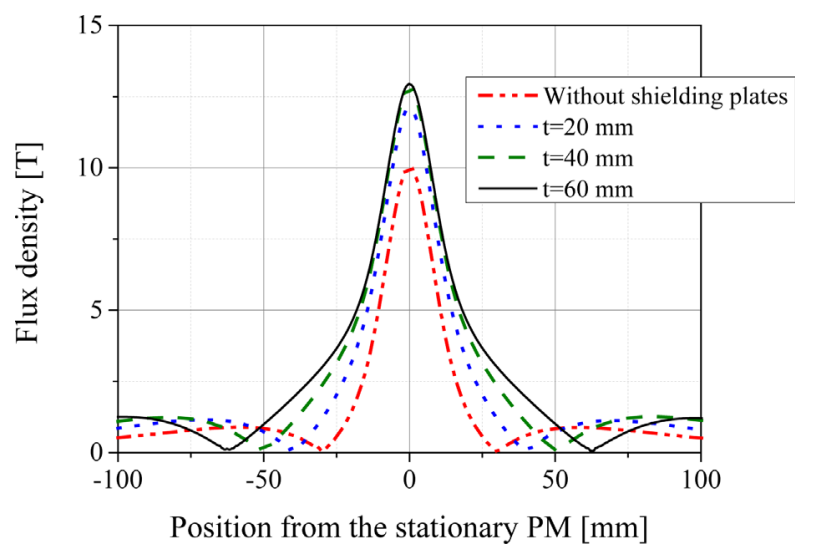

Fig. 8. (Color online) Resultant magnetic flux density at low limit position according to the thickness of magnetic shielding plates.

plates. It also shows convergence when the thickness of the plates is more than $40 \mathrm{~mm}$. Thus the thickness will be determined for design installation convenience at the detailed design stage.

\section{Conclusions}

The applicability of a magnetostrictive wire type RPIS on a nuclear reactor was analyzed. It is found out that the RPIS can measure the position of the control rods as well as identify the limit level of operation. The resolution could be enhanced with the optimized magnetic shielding 
plates. This research results will be the theoretical background for realizing precise rod position indication systems for a future nuclear reactor.

\section{Acknowledgements}

This work was supported by the National Research Foundation of Korea (NRF) grant funded by the Korea government (MSIP) (No. 2012M2A8A4026290).

\section{References}

[1] H. Huh, J. I. Kim, and K. J. Kim, Trans. of KIEE, 52D, (2003).

[2] C. A. Lawson and W. L. Dufek, Position indication system, Patent number 4371496, United States Patent, 1983.

[3] J. Joule, Annals of Electricity, Magnetism, and Chemistry
8, (1842).

[4] R. J. Krisst, Magnetostrictive position indicator, Patent number 4071818, United States Patent (1978).

[5] T. Ishida, S. Imayoshi, T. Yoritsune, H. Nunokawa, M.A. Ochiai, and Y. Ishizaka, J. Nuclear Sci. and Technol. 38, (2001).

[6] J. S. Lee, J. W. Kim, and K. K. Kim, Application number 10-2015-0022310, Korea Patent (2015).

[7] Pr. M. Drljaca, F. Vincent, P.-A. Besse, and R. S. Popovic, Sensors and Actuators, A 97-98 (2002).

[8] H. Blanchard, F. De Montmollin, J. Hubin, and R. S. Popovic, Sensors and Actuators, 82, (2000).

[9] User's Guide Maxwell [Online]. Available: http:// www.ansys.com, accessed (2015).

[10] J. Y. Yu, J. H. Kim, H. Huh, and J. I. Kim, Transactions of the Korean Nuclear Society Spring Meeting (2002).

[11] Data sheet-Terfenol-D, www.extrema.com/terfenol-d, Extrema Products Inc. 